\title{
ON GALOIS FIELDS OF CERTAIN TYPES*
}

\author{
BY \\ LEONARD CARLITZ
}

\section{INTRODUCTION}

Several writers have considered the relations between the $\zeta$-functions of an algebraic field and some of its subfields. Thus Artin $\dagger$ has, in a particular case, considered the question of the divisibility of the $\zeta$-function of a field by that of a subfield. In another paper $\ddagger$ he has shown how all possible $\zeta$-relations can be found. Explicit results of a general nature are however not arrived at. Herglotz $\S$ has investigated fields formed by the composition of several quadratic fields, thus generalizing a well known result of Dirichlet's. \ Pollaczek $\|$ has obtained results of a similar kind for Abelian fields with group of type $(1,1)$.

In all the cases cited, use is made of Hecke's** functional equation for the $\zeta$-function in an arbitrary field. Thus, for example, in Artin's first paper, a $\zeta$-relation is proved, except for a finite number of factors, by quite elementary methods; employing the functional equation, it is seen to hold in its entirety. Again, Herglotz and Pollaczek deduce discriminantal relationships by means of the functional equation.

In the following an explicit relation between $\zeta$-functions is deduced. The fields considered include as special cases those of Artin (first paper), Herglotz, and Pollaczek. No use is made of the Hecke functional equation; instead a method of an elementary nature is employed. Relations between discriminants also are easily proved by direct means. The first result of interest may be formulated thus:

Let $K$ be an (absolute) Galois field of degree $m$ and group $G_{m} . \dagger \dagger$ We make

* Presented to the Society, April 18, 1930; received by the editors in March, 1930.

$\dagger$ Ueber die Zetafunktionen gewisser algebraischer Zahlkörper, Mathematische Annalen, vol. 89 (1923), p. 147.

$\ddagger$ Ueber eine neue Art von L-Reihen, Abhandlungen aus dem Mathematischen Seminar der Hamburgischen Universität, vol. 3 (1924), p. 88.

\& Ueber einen Dirichletschen Satz, Mathematische Zeitschrift, vol. 12 (1922), p. 255.

Tा Werke, vol. 1, p. 533.

|| Ueber die Einheiten relativ-abelscher Zahlkörper, Mathematische Zeitschrift, vol. 30 (1929), p. 520.

** Ueber eine neue Anwendung der Zetafunktionen auf die Arithmetik der Zahlkörper, Göttinger Nachrichten, 1917, p. 90.

†† Hilbert,DieTheorie der algebraischen Zahlkörper, Jahresbericht der Deutschen MathematikerVereinigung, vol. 4 (1894-95), p. 248. 
the following assumptions concerning $G_{m}: G_{l}$ is an invariant subgroup of index $a, m=l a$. The complex $\left(G_{m}-G_{l}+l \cdot I\right), I$ representing the identity, may be exhibited as the sum of $l$ non-overlapping conjugate groups. Further let $k_{a}$ be the Galois field corresponding* to $G_{l}$. To the $l$ groups of order $a$ mentioned above, let there correspond the $l$ conjugate fields of degree $l, k_{l}, k_{l}^{\prime}, \cdots, k_{l}^{(l-1)}$. With the above definitions and assumptions we shall prove ( $\$ 3)$

$$
\zeta^{a} \zeta_{K}=\zeta_{k_{a}} \zeta_{k_{l}}{ }^{a} .
$$

If we next suppose that $K$ is a relative Galois field, the base field being some $F$, while all the remaining assumptions on $K$ are taken over for $K / F$, then (1) becomes

$$
\zeta_{F}^{a} \zeta_{K}=\zeta_{k_{a}} \zeta_{k_{l}}{ }^{a} .
$$

(It should be noted that all our $\zeta$-relations are proved only for a half-plane.) The second general result concerns the discriminants of the fields defined. If by $d(k)$ we denote the discriminant of any field $k$, we find $(\$ 4)$

$$
d(K)= \pm d\left(k_{a}\right) d^{a}\left(k_{l}\right),
$$

when $K$ is absolute Galoisian. In the more general case, we may suppose that each $d$ in (2) is a relative with respect to the field $F$. We then readily derive

$$
d^{a}(F) d(K)= \pm d\left(k_{a}\right) d^{a}\left(k_{l}\right),
$$

where each $d$ is now an absolute discriminant.

In $\$ 5$ are sketched the proof of formulas like (1) and (2) for the case of Abelian fields of type $(1,1, \ldots, 1)$; in particular might be mentioned (18) and (19).

In $\S \S 6,7,(1)$ and (2) and their analogs of $\S 5$ are applied to prove results that appear new. The results of the first section are contained in (30) and (31). A typical though particular result of $\$ 7$ follows:

Let $k_{4}{ }^{1}=k\left(\left[a+b^{1 / 2}\right]^{1 / 2}\right), k_{4}{ }^{2}=k\left(\left[2 a+2\left(a^{2}-b^{2}\right)^{1 / 2}\right]^{1 / 2}\right), k_{2}=k\left(\left[\left(a^{2}-b\right) b\right]^{1 / 2}\right)$, where neither $a^{2}-b$ nor $\left(a^{2}-b\right) b$ is a square. Then $K$, the field compounded of $k_{4}{ }^{2}, k_{4}{ }^{2}, k_{2}$ is readily seen to be Galoisian of degree eight, $\dagger$ and we shall prove

and

$$
\zeta^{2} \zeta_{K}=\zeta_{k_{2}} \zeta_{k_{4}} \zeta_{k_{i}^{*}}
$$

$$
d(K)= \pm d\left(k_{2}\right) d\left(k_{4}^{1}\right) d\left(k_{4}^{2}\right) \text {. }
$$

* Hilbert, loc. cit., p. 250.

† Seidelmann, Die Gesamtheit der kubischen und biquadratischen Gleichungen mit Affekt bei beliebigem Rationalitätsbereich, Mathematische Annalen, vol. 78 (1918), p. 232. 
By means of (1) and (2) a ratio of class numbers is transformed into a corresponding ratio of regulators. In $\$ 8$ this ratio of regulators is considered and, in a particular case, an upper and a lower bound of a simple sort are determined.

I wish to take this opportunity to acknowledge my indebtedness to Professor H. H. Mitchell for his valuable suggestions and his very helpful criticism.

\section{Properties of $G_{m}$}

Let $G_{a}$ be one of the $l$ subgroups of $G_{m}$ contained in $\left(G_{m}-G_{l}+l \cdot I\right)$. Its operators will be denoted by $T_{1}, \cdots, T_{a}$. The operators of $G_{l}$ will be taken as $S_{1}, \cdots, S_{l}$. We may suppose $S_{1}=T_{1}=I$.

\section{Lemima 1.}

$$
G_{m}=G_{l} \cdot T_{1}+\cdots+G_{l} \cdot T_{a}=G_{l} \cdot G_{a},
$$

and the factor group $G_{m} / G_{l}=G_{a}$.

To prove this, we observe first that the number of elements in $G_{l} \cdot T_{1}+\cdots+G_{l} \cdot T_{a}$ is $l a=m$. Further, no two complexes $G_{l} T_{\alpha}, G_{l} T_{\beta}$ have an element in common. For suppose

then

$$
S_{i} T_{\alpha}=S_{j} T_{\beta} \quad\left(S_{i}, S_{j} \text { in } G_{l}\right) ;
$$

$$
S_{j}^{-1} S_{i}=T_{\beta} T_{\alpha}^{-1} .
$$

But $G_{l}$ and $G_{a}$ have only $I$ in common; accordingly

$$
S_{i}=S_{i} \text { and } T_{\alpha}=T_{\beta},
$$

which shows that all the elements in the sum are distinct.

\section{LEMMA 2.}

$$
l \equiv 1
$$

$(\bmod a)$

1. Let $S_{i}(2 \leqq i \leqq l)$ be any element of $\left(G_{l}-I\right)$. Then the set $T_{\alpha} S_{i} T_{\alpha}^{-1}$ $(\alpha=1, \cdots, a)$ consists of $a$ distinct elements of $G$. For from $T_{\alpha} S_{i} T_{\alpha}^{-1}=T_{\beta} S_{i} T_{\bar{\beta}}^{-1}$ we have $S_{i} T_{\alpha}^{-1} T_{\beta} S_{i}^{-1}=T_{\alpha}^{-1} T_{\beta}$. But this implies $T_{\alpha}=T_{\beta}$.

2. Let now $S_{j}$ be any element of $\left(G_{l}-I\right)$ not in the set $T_{\alpha} S_{i} T_{\alpha}^{-1}$. Then the sets $T_{\alpha} S_{i} T_{\alpha}^{-1}$ and $T_{\alpha} S_{j} T_{\alpha}^{-1}$ have no element in common. For from $T_{\alpha} S_{i} T_{\alpha}^{-1}=T_{\beta} S_{j} T_{\beta}^{-1}$ we should have $S_{j}=T_{\bar{\beta}}^{-1} T_{\alpha} S_{i} T_{\alpha}^{-1} T_{\beta}$, which would mean that $S_{j}$ belongs to the set $T_{\alpha} S_{i} T_{\alpha}^{-1}$.

We see then that the $l-1$ elements $S_{i}(i=2, \cdots, l)$ fall into sets each containing $a$ elements, and that no two of these sets have an element in common. Hence the truth of (4). 
Suppose now that $G_{M}$ is any subgroup of $G_{m}$. Let

$$
G_{L}=D\left(G_{M}, G_{l}\right),
$$

that is, the group common to $G_{M}$ and $G_{l}$. We easily recognize that $G_{L}$ is invariant under $G_{M}$. The nature of the factor group $G_{M} / G_{L}$ is described by Lemmas 3 and 4.

LEMMA 3. The factor group $G_{M} / G_{L}$ is isomorphic with a subgroup of $G_{a}$.

The lemma follows immediately from a known theorem.*

LEMMA 4. The factor group $G_{M} / G_{L}$ is isomorphic with $G_{A}$, a subgroup of $G_{M}$ having only the identity in common with $G_{L}$.

If $G_{L}$ does not exhaust $G_{M}$ let us take as $G_{a}$ one of the $l$ isomorphic subgroups that has in common with $G_{M}$ at least one element other than the identity.

By (3), $G_{m}=G_{l} \cdot T_{1}+\cdots+G_{l} \cdot T_{a} ; G_{a}=\left(T_{1} \cdots T_{a}\right)$. Let us suppose that $G_{M}$ is contained in the first $A$ complexes $G_{l} \cdot T_{1}+\cdots+G_{l} \cdot T_{A}$ and that the $T$ 's are so numbered that each complex actually contains at least one element of $G_{M}$. Comparison with the proof of Lemma 3 shows that the complex $\left(T_{1} \cdots T_{A}\right)$ forms a subgroup $G_{A}$ of $G_{M}$ isomorphic with $G_{M} / G_{L}$, thus establishing the lemma.

From Lemma 4 follows without difficulty

LEMMA 5. The complex $\left(G_{M}-G_{L}+L \cdot I\right)$ may be exhibited as the sum of $L$ non-overlapping conjugate groups each isomorphic with $G_{M} / G_{L}$.

LEMMA 6. Let $G_{M}$ be an invariant subgroup of $G_{m}$, such that $G_{m} / G_{M}$ is cyclic of order $\mu$. Then, either

$$
\begin{aligned}
& l=L \text { and } a=\mu A ; \text { or } \\
& l=\mu L \text { and } a=A=1 .
\end{aligned}
$$

1. Assume (I) does not hold, and that $A>1$.

Now the $L$ subgroups $G_{A}$ of $G_{M}$ are contained in $L$ of the $l$ subgroups $G_{a}$. Let $S$ (of $G_{l}$ ) transform one of these last groups into a $G_{a}$ having only the identity in common with the set of $L$ groups. Then the $G_{A}$ it contains is transformed into a group necessarily different from any of the $L$ subgroups $G_{A}$ contained in $G_{M}$. Hence, when $l>L$ and $A>1, G_{M}$ cannot be invariant under $G_{m}$.

2. Suppose then that $l>L, A=1$, but $a>A$.

Then

* Cf. Fricke, Algebra, 1924, vol. 1, p. 277. 
(6)

$$
\begin{aligned}
G_{m}=G_{l} G_{a} & =\sum_{i=1}^{1 / L} \sum_{j=1}^{a} S_{i} G_{L} T_{j} \\
& =\sum \sum S_{j} G_{M} T_{j}=\sum \sum G_{M} S_{i} T_{j} \\
& =\sum_{i=0}^{\mu-1} G_{M} V^{2} .
\end{aligned}
$$

But $V=S T(T \neq I)$ is an element of $\left(G_{m}-G_{l}\right)$ and therefore of order $\leqq a$. But $\mu=l a / L>a$; (6) is then impossible.

\section{Derivation of the $\zeta$-Relation}

Dedekind* has shown how to derive the decomposition of a rational prime in a subfield of a Galois field when its decomposition in the larger field is assumed:

Let $K$ be a Galois field of degree $m$ and group $H_{m}$. Let $k$ be a subfield of $K$ of degree $\mu$ corresponding to a subgroup $H_{\nu}$ of order $\nu, m=\mu \nu$. Suppose that in $K$

$$
p=\left(\mathfrak{B}_{1} \cdots \mathfrak{B}_{e}\right)^{\lambda} ; \mathfrak{B}_{i} \text { of degree } g
$$

Then in $k$,

$$
p=p_{1}^{a_{1}} \cdots p_{\tau}^{a_{\tau}} ; p_{i} \text { of degree } g_{i}^{\prime}
$$

and

$$
\mathfrak{p}_{i}=\left(\mathfrak{B}_{i 1} \cdots \mathfrak{P}_{i \rho_{i}}\right)^{\lambda_{i}} ; \mathfrak{P}_{i j} \text { of relative degree } g_{i} \text {. }
$$

The quantities $\tau, a_{i}, \rho_{i}, g_{i}, g_{i}^{\prime}, \lambda_{i}$ must now be determined.

Let $H_{k}$ be the "Zerlegungsgruppe" $\dagger$ of any prime ideal $\mathfrak{P}$ in the right member of (7). Let $H_{m}$ be decomposed with respect to the two subgroups $H_{\nu}, H_{\kappa}$ :

$$
H_{m}=H_{\nu} \cdot V_{1} \cdot H_{k}+\cdots+H_{\nu} \cdot V_{\tau} \cdot H_{k}
$$

the number of complexes is precisely $\tau$; no two complexes have an element in common; the number of elements in the $i$ th complex is $\kappa \rho_{i}$, where $\sigma_{i} \rho_{i}=\nu$ and

$$
H_{\sigma_{i}}=D\left(V_{i} \cdot H_{k} \cdot V_{i}^{-1}, H_{v}\right) \text {. }
$$

If $H_{\lambda}$ denote the "Trägheitsgruppe" $\ddagger$ of the ideal $\mathfrak{B}$, then $\lambda_{i}$ is the order of the group

* Zur Theorie der Ideale, Göttinger Nachrichten, 1894, p. 272; or Fricke, Algebra, 1928, vol. 3, p. 186.

† Cf. Fricke, loc. cit., p. 171.

$\ddagger$ Fricke, loc. cit. 


$$
H_{\lambda_{i}}=D\left(V_{i} \cdot H_{\lambda} \cdot V_{i}^{-1}, H_{v}\right) \text {. }
$$

Other relationships to be noted are

$$
\kappa=g \lambda, \quad g=g_{i} g_{i}^{\prime}, \quad g_{i} \lambda_{i}=\sigma_{i}, \quad a_{i} \lambda_{i}=\lambda, \quad \sum \rho_{i}=e .
$$

To apply the Dedekind theory, suppose $H_{m} \equiv G_{m}$ as defined above.

1. Let $H_{\nu}=G_{l}$ :

$$
G_{m}=G_{l} \cdot V_{1} \cdot H_{k}+\cdots+G_{l} \cdot V_{\tau} \cdot H_{k} .
$$

By Lemma 5, $H_{\kappa}=G_{L} G_{A}, G_{L}=D\left(H_{k}, G_{l}\right)$; and since $H_{\kappa}$ satisfies all the assumptions made on $G_{m}$, we have $H_{\lambda}=G_{L^{\prime}} \cdot G_{A^{\prime}}$, where

$$
G_{L^{\prime}}=D\left(G_{L}, H_{\lambda}\right), H_{\lambda} / G_{L^{\prime}}=G_{A^{\prime}}
$$

We have then

and

$$
\begin{aligned}
& D\left(V_{i} \cdot H_{k} \cdot V_{i}^{-1}, G_{l}\right)=V_{i} \cdot G_{L} \cdot V_{i}^{-1}, \\
& D\left(V_{i} \cdot H_{\lambda} \cdot V_{i}^{-1}, G_{l}\right)=V_{i} \cdot G_{L^{\prime}} \cdot V_{i}^{-1} .
\end{aligned}
$$

Therefore,

$$
\begin{array}{llll}
\sigma_{i}=L, & \rho_{i}=l / L, & \tau \rho_{i}=e, & \tau=e L / l \\
\lambda_{i}=L^{\prime}, & g_{i}=L / L^{\prime}, & g_{i}^{\prime}=g L^{\prime} / L, & a_{i}=\lambda / L^{\prime} .
\end{array}
$$

2. Let $H_{\nu}=G_{a}$ :

$$
G_{m}=G_{a} \cdot V_{1} \cdot H_{\kappa}+\cdots+G_{a} \cdot V_{\tau} \cdot H_{k} .
$$

We may suppose $G_{a}$ is that one of the $l$ conjugates that contains $G_{A}(A>1$; when $A=1$, any $G_{a}$ will serve the purpose). Evidently

$$
D\left(V_{i} \cdot H_{k} \cdot V_{i}^{-1}, G_{a}\right)=\left\{\begin{array}{l}
G_{A^{\prime}} \text { for } i=1 \\
G_{1} \text { for } i>1
\end{array}\right.
$$

whence

$$
\begin{gathered}
\sigma_{1}=A, \quad \sigma_{2}=\cdots=\sigma_{\tau}=1 ; \\
\rho_{1}=\frac{a}{A}, \quad \rho_{2}=\cdots=\rho_{\tau}=a ; \\
D\left(V_{i} \cdot H_{\lambda} \cdot V_{i}^{-1}, G_{a}\right)=\left\{\begin{array}{l}
G_{A} \text { for } i=1, \\
G_{1} \text { for } i>1 ;
\end{array}\right. \\
\lambda_{1}=A^{\prime}, \quad \lambda_{2}=\cdots=\lambda_{\tau}=1 ; \quad g_{1}=\frac{A}{A^{\prime}}, \quad g_{2}=\cdots=g_{\tau}=1 ; \\
g_{1}^{\prime}=\frac{g A^{\prime}}{A}, \quad g_{2}^{\prime}=\cdots=g_{\tau}^{\prime}=g ; \quad a_{1}=\frac{\lambda}{A^{\prime}}, \quad a_{2}=\cdots=a_{\tau}=\lambda ;
\end{gathered}
$$




$$
\frac{a}{A}+(\tau-1) a=e ; \tau-1=\frac{e}{a}-\frac{1}{A} .
$$

Now in $K$ we clearly have, using (7),

$$
\zeta_{K}(s)=\prod_{p} \frac{1}{\left(1-\frac{1}{p^{o s}}\right)^{e}} .
$$

Let us fix our attention on a particular $G_{\kappa}$, and suppose it the Zerlegungsgruppe for some $\mathfrak{B}, \mathfrak{B} / p$. We then seek that factor of $\zeta_{k_{a}}(s)$ corresponding to $p$. By (8), the decomposition of $p$ in $k_{a}$ is

$$
p=\left(p_{1} \cdots p_{\tau}\right)^{\lambda / L^{\prime}}, \tau=\frac{e L}{l} ;
$$

each $\mathfrak{p}$ of degree $g L^{\prime} / L$. Therefore the factor in question is

$$
\frac{1}{\left(1-\frac{1}{p^{o L^{\prime} / L}}\right)^{e L / L}} .
$$

As for the factor in $k_{l}$, we have, by (9), that $p$ decomposes in $k_{l}$ thus:

$$
p=\mathfrak{p}_{1}^{\lambda / A^{\prime}}\left(\mathfrak{p}_{2} \cdots \mathfrak{p}_{\tau}\right)^{\lambda}, \tau=1+\frac{e}{a}-\frac{1}{A}
$$

degree of $\mathfrak{p}_{1}=g A^{\prime} / A$; degree of $\mathfrak{p}_{i}(i=2, \ldots, \tau)=g$. We find then

$$
\frac{1}{\left(1-\frac{1}{p^{g A^{\prime} s / A}}\right)\left(1-\frac{1}{p^{g s}}\right)^{e / a-1 / A}} \text {. }
$$

To prove (1) we must then verify that the product of the expression (11) by the $a$ th power of (12) is (using (10))

$$
\frac{1}{\left(1-\frac{1}{p^{08}}\right)^{e}} \frac{1}{\left(1-\frac{1}{p^{8}}\right)^{a}}
$$

We notice first that $G_{k}$, necessarily a subgroup of $G_{m}$, has the same properties as $G_{m}$ (Lemma 5). Further it is known that $G_{\lambda}$ is invariant under $G_{\kappa}$ and that the factor group $G_{\kappa} / G_{\lambda}$ is cyclic.* Lemma 6 may then be applied,

* Fricke, loc. cit., p. 175. 
and we find that either

$$
\begin{aligned}
& L=L^{\prime} \text { and } A=g A^{\prime} ; \text { or } \\
& L=g L \text { and } A=A^{\prime}=1 .
\end{aligned}
$$

(I) We must show that

$$
\left(1-\frac{1}{p^{08}}\right)^{-e L / l}\left[\left(1-\frac{1}{p^{8}}\right)\left(1-\frac{1}{p^{08}}\right)^{e / a-1 / A}\right]^{-a}=\left(1-\frac{1}{p^{08}}\right)^{-e}\left(1-\frac{1}{p^{8}}\right)^{-a} ;
$$

which is correct, since

$$
\frac{e L}{l}+e-\frac{a}{A}=e+\frac{e L A-l a}{l A}=e+\frac{e \kappa-m}{l A}=e .
$$

(II) Here we are to prove

$$
\left(1-\frac{1}{p^{\circ}}\right)^{-e L / l}\left[\left(1-\frac{1}{p^{00}}\right)^{e / a}\right]^{-a}=\left(1-\frac{1}{p^{0 s}}\right)^{-e}\left(1-\frac{1}{p^{0}}\right)^{-a} .
$$

This follows immediately from

$$
\frac{e L}{l}=\frac{e L A}{l a} \cdot a=\frac{e k}{m} \cdot a=a .
$$

The proof of (1) is then complete.

It is unnecessary to give the proof of (1a) since the Dedekind theory holds (with one obvious modification) for relative Galois fields.*

\section{Proof of (2)}

Let $\Omega$ be the fundamental form $\dagger$ of $K$, and let $\delta$ be the "differente" of $K$. Evidently

$$
\delta=\prod_{\substack{U \text { in } G_{m} \\ U \neq I}}(\Omega-U \Omega) .
$$

If $k$ be any subfield of $K$, we shall let $\delta(k)$ and $\mathfrak{D}(k)$ denote respectively the differente of $k$ and the differente of $K$ relative to $k$. As is well known $\ddagger$

$$
\delta=\delta(k) \mathfrak{D}(k) \text {. }
$$

If we suppose that $k$ corresponds to the subgroup $G_{M}$ of $G_{m}$, then

$$
\mathfrak{D}(k)=\prod_{\substack{U \text { in } G_{M} \\ U \neq I}}(\Omega-U \Omega) .
$$

* See, for example, Weber, Algebra, 1899, vol. 2, p. 657.

† Cf. Hilbert, loc. cit., p. 195.

$\ddagger$ Hilbert, loc. cit., Satz 41 . 
Applying (15) we find that

$$
\begin{aligned}
& \mathfrak{D}\left(k_{a}\right)=\Pi(\Omega-U \Omega), U \text { in }\left(G_{l}-I\right), \\
& \mathfrak{D}\left(k_{l}\right)=\Pi(\Omega-U \Omega), U \text { in }\left(G_{a}-I\right) \text {, } \\
& \mathfrak{D}\left(S_{2} k_{l}\right)=\Pi(\Omega-U \Omega), U \text { in }\left(S_{2} G_{a} S_{2}^{-1}-I\right) \text {, } \\
& \mathfrak{D}\left(S_{l} k_{l}\right)=\Pi(\Omega-U \Omega), \quad U \text { in }\left(S_{l} G_{a} S_{l}^{-1}-I\right) .
\end{aligned}
$$

Multiplying together the corresponding members of these $l+1$ equations we get (using (13))

$$
\mathfrak{D}=\mathfrak{D}\left(k_{a}\right) \mathfrak{D}\left(k_{l}\right) \mathfrak{D}\left(S_{2} k_{l}\right) \cdots \mathfrak{D}\left(S_{l} k_{l}\right)
$$

or, by (14),

$$
\mathfrak{d}^{l}=\mathfrak{d}\left(k_{a}\right) \mathfrak{d}\left(k_{l}\right) \mathfrak{d}\left(S_{2} k_{l}\right) \cdot \cdots \mathfrak{d}\left(S_{l} k_{l}\right) .
$$

But

$$
d\left(k_{l}\right)=\mathfrak{d}\left(k_{l}\right) \mathfrak{b}\left(S_{2} k_{l}\right) \cdots \mathfrak{d}\left(S_{l} k_{l}\right), *
$$

so that (16) may be written

$$
\mathfrak{d}^{l}=\mathfrak{d}\left(k_{a}\right) d\left(k_{\imath}\right) .
$$

Now in a Galois field (of degree $m$ ) the discriminant is the $m$ th power of the differente. $\dagger$ Hence, from (17) we infer

$$
d=d\left(k_{a}\right) d^{a}\left(k_{l}\right) .
$$

It seems scarcely necessary to go into the proof of (2a); the remark made in the Introduction indicates how it may be readily derived from (2).

\section{Abelian Fields of type $(1,1, \ldots, 1)$}

We shall now briefly consider Abelian fields that are compounded of cyclic fields of equal prime degree. Assume then $K$ an Abelian field with group $G_{q^{f}}$ of type $\left(1,1, \ldots\right.$, to $f$ units), $q$ a prime. $G_{q}$ contains $\left(q^{f}-1\right) /(q-1)$ subgroups of order $q^{f-1} ; \ddagger$ hence $K$ contains $\left(q^{f}-1\right) /(q-1)$ cyclic fields. If these fields be denoted by $k^{1}, \ldots, k^{r_{f}}$, where $r_{f}=\left(q^{f}-1\right) /(q-1)$, then we shall prove

$$
\begin{gathered}
\zeta^{r j-1} \zeta_{K}=\zeta_{K^{1}} \cdots \zeta_{K^{r}} \\
d(K)=d\left(k^{1}\right) \cdots d\left(k^{r}\right) . \S
\end{gathered}
$$

* Hilbert, loc. cit., Satz 37.

$\dagger$ Hilbert, loc. cit., Satz 68.

$\ddagger$ Cf. Burnside, Theory of Groups, 1912, p. 60.

\& Cf. Pollaczek, loc. cit., p. 534. 
(I) Proof of (18). Assume that in $K, p=\left(\mathfrak{P}_{1} \ldots \mathfrak{P}_{e}\right)^{\lambda}$, each $\mathfrak{P}$ of degree g. We employ Dedekind's method ( $\$ 3)$. Let $G_{x}$ be the Zerlegungsgruppe of $\mathfrak{P}_{1}$ (and therefore of each of the remaining $\mathfrak{P}^{\prime}$ s). Since $G_{x} / G_{\lambda}$ is cyclic, there are but two cases to consider: (1) $\kappa=\lambda$; (2) $\kappa=q \lambda$.

(1) $\kappa=\lambda=q^{t}$. Put $G_{q f}=G_{\nu} \cdot V_{1} \cdot G_{\kappa}+\ldots+G_{\nu} \cdot V_{\tau} \cdot G_{\kappa}$, where $G_{\nu}$ is one of the $r_{f}$ subgroups of $G_{q f}$ of order $q$. (Corresponding to $G_{\nu}$ is then a field of degree $q^{f-1}$.)

Now

$$
\begin{aligned}
D\left(G_{x}, G_{\nu}\right) & =\left\{\begin{array}{l}
G_{q} \text { for } r_{t} \text { choices of } G_{v}, \\
G_{1} \text { for }\left(r_{f}-r_{t}\right) \text { choices of } G_{\nu} ;
\end{array}\right. \\
D\left(G_{\lambda}, G_{\nu}\right) & =D\left(G_{k}, G_{v}\right) .
\end{aligned}
$$

For $r_{t}$ choices of $G_{v}$, then, $\tau=e, g_{i}^{\prime}=1$; for the remaining $\left(r f-r_{t}\right)$ choices, $\tau=e / q, g_{i}^{\prime}=1$. Typical factors of the associated $\zeta$-functions are then

Since

$$
\frac{1}{\left(1-\frac{1}{p^{s}}\right)^{e}} \text { and } \frac{1}{\left(1-\frac{1}{p^{s}}\right)^{e / p}} \text {, respectively. }
$$

$$
e r_{t}+e\left(r_{f}-r_{t}\right) / q=e r_{f-1}+q^{f-1},
$$

we may conclude that in this case $(\kappa=\lambda)$

$$
\begin{aligned}
& \left(\Pi \frac{1}{\left(1-\frac{1}{p^{s}}\right)^{c}}\right)^{r_{t}}\left(\Pi \frac{1}{\left(1-\frac{1}{p^{d}}\right)^{c / q}}\right)^{f /-r_{t}} \\
& =\left(\Pi \frac{1}{\left(1-\frac{1}{p^{s}}\right)^{c}}\right)^{r_{f-1}}\left(\Pi \frac{1}{1-\frac{1}{p^{s}}}\right)^{q f-1},
\end{aligned}
$$

the products extending only to primes $p$ for which $\kappa=\lambda$.

(2) $\kappa=q^{t}, \lambda=q^{t-1}$. Again put $G_{q}=G_{v} \cdot V_{1} \cdot G_{\kappa}+\ldots+G_{v} \cdot V_{1} \cdot G_{k}$. Then $D\left(G_{k}, G_{v}\right)=G_{q}$ for $r_{t}$ choices of $G_{v}$; and for these choices of $G_{v}$,

$$
D\left(G_{\lambda}, G_{\nu}\right)=\left\{\begin{array}{l}
G_{q} r_{t-1} \text { times; } \\
G_{1} \text { the remaining } q^{t-1} \text { times. }
\end{array}\right.
$$

$D\left(G_{k}, G_{v}\right)=G_{1}$ for $\left(r_{f}-r_{t}\right)$ choices of $G_{v}$; and for each such choice $D\left(G_{\lambda}, G_{\nu}\right)=G_{1}$.

Therefore,

$$
\begin{aligned}
& \text { for } r_{t-1} \text { choices of } G_{v}, \tau=e, g_{i}^{\prime}=q ; \\
& \text { for } q^{t-1} \text { choices of } G_{v}, \tau=e, g_{i}^{\prime}=1 ;
\end{aligned}
$$




$$
\text { for }\left(r_{f}-r_{t}\right) \text { choices of } G_{\nu}, \tau=e / q, g_{i}^{\prime}=q \text {. }
$$

Factors of the corresponding $\zeta$ 's are then

$$
\frac{1}{\left(1-\frac{1}{p^{q s}}\right)^{e}}, \frac{1}{\left(1-\frac{1}{p^{8}}\right)^{c}} \text { and } \frac{1}{\left(1-\frac{1}{p^{q^{8}}}\right)^{8 / q}} \text {, respectively. }
$$

From this we may write, since $e \cdot q^{t-1}=q^{f-1}$, and

$$
\begin{gathered}
e r_{t-1}+\left(r_{f}-r_{t}\right) e / q=e r^{f-1} \\
\left(\Pi\left(1-\frac{1}{p^{q s}}\right)^{-e}\right)^{r_{t-1}}\left(\Pi\left(1-\frac{1}{p^{q s}}\right)^{-e / q}\right)^{r_{f-r t}}\left(\Pi\left(1-\frac{1}{p^{s}}\right)^{-e}\right)^{q t-1} \\
=\left(\Pi\left(1-\frac{1}{p^{q s}}\right)^{-e}\right)^{r_{f-1}}\left(\Pi\left(1-\frac{1}{p^{s}}\right)^{-1}\right)^{q^{f-1}},
\end{gathered}
$$

the products extending only to primes $p$ for which $\kappa=q \lambda$.

From (20) and (21) follows immediately

$$
\zeta^{q f-1} \zeta_{K}=\prod_{k} \zeta_{k}
$$

the product extending over the $r_{f}$ fields of degree $q^{f-1}$. Making use of (22), (18) follows easily by induction on $f$.

(II) Proof of (19). We notice, to begin with, that

$$
\left(G_{q f}-I\right)=\sum\left(G_{q}-I\right),
$$

the sum extending over the $r_{f}$ subgroups $G_{q}$ of $G_{q}$.

Then, if $\mathfrak{D}(k)$ and $\mathfrak{D}(k)$ have the same significance as in $\$ 4$,

$$
\mathfrak{D}=\mathfrak{D}(K)=\prod_{k} \mathfrak{D}(k),
$$

the product extending over the $r_{f}$ subfields of $K$ of degree $q^{f-1}$. Equations (23) together with $\delta=\delta(k) \mathfrak{D}(k)$ imply

$$
\delta^{r-1}=\prod_{1}^{r y} \delta(k) .
$$

Raising both members of this equality to the $q^{f-1}$ th power, we find

$$
d^{r_{f-1}}(K)= \pm \prod_{1}^{\prime f} d(k), k \text { of degree } q^{f-1}
$$

Then, exactly as in proving (18), we may prove (19) by induction, making use of (24). 
We may put (19) in a more general form that will be seen to include (24) as well. The proof consists of an easy induction so that we shall state the result only:

$$
d^{r-1, m-1}(K)= \pm \prod_{1}^{\tau^{\prime} m} d(k)
$$

where the product extends over all the subfields of $K$ of degree $q^{m}$, and $r_{f, m}$ is the number of subgroups of $G_{q} f$ of order $q^{m}$ :

$$
r_{f, m}=\frac{q^{f}-1}{q-1} \cdots \frac{q^{f-m+1}-1}{q^{m}-1} .
$$

A similar generalization holds for (18):

$$
\zeta^{r /, m-r_{f-1, m-1}} \zeta_{K}^{r-1, m-1}=\prod_{1}^{r / m} \zeta_{k},
$$

the product being taken as in (25).

Finally, if we suppose $K$ relative Abelian, the base field being some $F$, while everything else remains unchanged, then we may rewrite (25) and (26) thus:

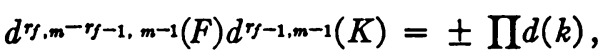

$$
\begin{aligned}
& \zeta_{F}^{r, m^{-r} f-1, m^{-1}} \zeta_{K}^{r j-1, m-1}=\Pi \zeta_{k},
\end{aligned}
$$

the products being taken as in (25).

\section{Several ILlustrations AND AN APPLICATION}

It may be of interest at this point to give several examples illustrating the group $G_{m}$ defined in $\$ 1$.

(I) We mention first the case treated by Artin.* The group $G_{m}$ may be defined as a group of transformations

$$
x^{\prime}=\alpha x+\beta,
$$

$\alpha$ and $\beta$ being marks of a finite (Galois) field of degree $l=q^{f}$. The subgroup $G_{l}$ consists of the transformations

$$
x^{\prime}=x+\beta
$$

a particular $G_{a}$ is defined by $x^{\prime}=\alpha x$, so that it is in this case cyclic.

(II) In this example, $G_{l}$ and $G_{a}$ are Abelian but $l$ may be divisible by more than one prime.

- Mathematische Annalen, vol. 89 (1923), p. 147. 
Let $l=p_{1}{ }^{e_{1}} \cdots p_{r}{ }^{\text {er }}\left(e_{i} \geqq 1\right)$; let $a$ be chosen so that $p_{i} \equiv 1$, mod $a$; let $u_{i}$ appertain to $a, \bmod p_{i}{ }^{6} ;$ choose $u$ to satisfy $u \equiv u_{i}, \bmod p_{i}{ }^{6 i} ;$ then we define $G_{m}$ as the group generated by $S$ and $T, S_{i}=I=T^{a}, T S T^{-1}=S^{u}$. The group $G_{l}$ is generated by $S$; and $G_{a}$ may be taken as $T$ and its powers.

(III) For the third instance, ${ }^{*}$ we use a subgroup $G_{72}$ of the Hessian group $G_{216 .}$

The $G_{72}$ is generated by $S_{1}, S_{2}, T_{1}, T_{2}$, where

$$
\begin{gathered}
S_{1}^{8}=S_{2}^{8}=I, \quad S_{2} S_{1}=S_{1} S_{2} ; \\
T_{1}^{4}=T_{2}^{4}=I, \quad T_{1}^{2}=T_{2}^{2} \neq I, \quad T_{2} T_{1} \neq T_{1}^{8} T_{2} ; \\
T_{1} S_{1} T_{1}^{-1}=S_{1} S_{2}, \quad T_{1} S_{2} T_{1}^{-1}=S_{1} S_{2}^{2}, \\
\quad T_{2} S_{1} T_{2}^{-1}=S_{1}^{2} S_{2}, \quad T_{2} S_{2} T_{2}^{-1}=S_{1} S_{2} .
\end{gathered}
$$

$G_{l}$ consists of $S_{1}, S_{2}$, and products of powers of these elements; $G_{l}$ is evidently Abelian of type $(1,1)$.

For a particular $G_{a}$ we take the group of order eight generated by $T_{1}$ and $T_{2}$. This group is not Abelian; it is indeed the quaternion group.

Returning to the first example, let $K$ be a Galois field with group $G_{l a}$ as described $\left(l=q^{f}\right)$. Let $k_{a}$ be the field corresponding to $G_{l}$; it is evidently Abelian. Let $k_{l}$ be one of the $l$ conjugate fields of degree $l$. Suppose it possible to choose $m$ so that $0<m<f, q^{m} \equiv 1, \bmod a$. Then it is easily seen that any subfield of $K$ of degree $q^{m} a$ is Galoisian; furthermore its structure is exactly like that of $K$ if we merely replace $f$ by $m$.

To begin with, let us think of $K$ as relative Galoisian to $k_{a}$; the relative group is Abelian of type $(1,1, \ldots, 1)$. By (26a), if $F \equiv k_{a}$,

$$
\zeta_{k_{a}}^{r j, m-r j-1, m-1} \zeta_{K}^{r j-1, m-1}=\prod_{1}^{r j, m} \zeta_{k},
$$

the product extending over all subfields of $K$ that are of degree $q^{m}$ relative to $k_{a}$ (that is, to $F$ ). Now, by (1),

$$
\zeta^{a} \zeta_{K}=\zeta_{k_{a}} \zeta_{k_{l}}^{a}
$$

and $k_{j}^{\prime}$ denoting one of the subfields of degree $j=q^{m}$ of some $k(k$ as in (27)),

$$
\zeta^{a} \zeta_{k}=\zeta_{k_{a}} \zeta_{k_{j}}
$$

for each $k$. Substituting (28) and (29) in (27), we get

$$
\zeta^{r, m-r /-1, m-1} \zeta_{k_{l}}^{r j-1, m-1}=\prod_{1}^{r_{j}, m} \zeta_{k_{j}}
$$

\footnotetext{
* This was suggested by Professor Mitchell.
} 
the product on the right extending over all fields of degree $j=q^{m}$ (each set of $j$ conjugate fields but one representative, namely, the one contained in $k_{l}$ ).

We may in exactly the same way derive a relation like (30) for discriminants:

$$
d\left(k_{l}\right)^{r-1, m-1}= \pm \prod_{1}^{r j, m} d\left(k_{j}\right) .
$$

\section{A SECOND APPLICATION}

We consider again the group $G_{m}$ of $\S 1$, but we shall now make an additional restriction: $a$ is some prime, $q$. Let us define a group $G_{l q^{2}}$ of order $l q^{2}$ as the direct product of $G_{m}$ and $H_{q}$, a group of order $q$. The group $G_{l^{2}}$ then contains $q$ invariant subgroups isomorphic with $G_{m}$ : If $G_{q}$ be generated by $T$, i.e., $G_{q}=\{T\}$, and $H_{q}=\{U\}$, then the $q$ subgroups are

$$
G_{l} \cdot\left\{U^{i} T\right\} \quad(i=1, \cdots, q) .
$$

Suppose now $K$ is an absolute Galois field with group $G_{l q^{2}}$ as defined above. To each $G_{l} \quad\left\{U^{i} T\right\}$ corresponds a cyclic field $k_{q}^{i}$ of degree $q ; K$ is a relative Galois field of relative degree $m$ with respect to $k_{q}^{i}$; the relative group is $G_{l} \cdot\left\{U^{i} T\right\}$, i.e. $G_{m}$. Accordingly, if in (1a) we let $F \equiv k_{q}^{i}$,

$$
\zeta_{k_{q}^{i}}^{q} \zeta_{K}=\zeta_{K_{q}^{i}}^{i} \zeta_{K_{l}^{i}}^{q} \quad(i=1, \cdots, q),
$$

where $K_{q}^{i}$ is of relative degree $q$, and $K_{l}^{i}$ is of relative degree $l$. The field $K_{q}^{i}$ is then of absolute degree $q$; if we think of $K$ as an absolute Galois field, then, since $G_{l q^{2}}$ has but one subgroup of order $l$, evidently all the symbols $K_{q}^{i}$ denote the same field, $K_{q}$, say. Further $G_{l}$ is invariant under $G_{l_{q} q^{2}}$ and the factor group is Abelian of type $(1,1)$; therefore $K_{q}$ is Abelian with group of the same type. The $q$ fields $k_{q}^{i}$ are of course subfields of $K_{q}$; it must contain one other; namely, the field corresponding to the subgroup $G_{l} \times H_{q}(\times$ means direct product); this field will be called $k_{q}$. By (18),

$$
\zeta^{q} \zeta_{K_{q}}=\zeta_{k_{q}} \prod_{i=1}^{q} \zeta_{k_{q}^{i}} \text {. }
$$

Multiplying together the $q$ equations (32), we find

$$
\zeta_{K} \prod_{i=1}^{q} \zeta_{k_{q}^{i}}=\zeta_{K_{q}} \prod_{i=1}^{q} \zeta_{K_{i}^{i}}
$$

Applying (33), this may be written

$$
\zeta^{q} \zeta_{K}=\zeta_{k_{q}} \prod_{i=1}^{q} \zeta_{K_{l}}
$$


For the discriminants we prove without any change in method

$$
d^{q}\left(k_{q}^{i}\right) d(K)= \pm d\left(K_{q}\right) d^{q}\left(K_{l}^{i}\right),
$$

and

$$
d(K)= \pm d\left(k_{q}\right) \Pi d\left(K_{l}^{i}\right),
$$

corresponding to (32) and (34) respectively.

The special case mentioned in the Introduction does not fall under the above, but may be handled in much the same way. The group of $K$ (using the notation of $\$ 1$ ) is $G_{8}$ :

$$
G_{8}=\{S, T\}, \quad S^{4}=I=T^{2}, \quad T S T=S^{-1} .
$$

To $\{T\}$ corresponds $k_{4}{ }^{1}$; to $\{S T\}, k_{4}^{2}$; to $\{S\}, k_{2}$. Let $k_{2}{ }^{2}, k_{2}{ }^{2}$ correspond to $\left\{S^{2}, T\right\},\left\{S^{2}, S T\right\}$, respectively. To the invariant subgroup $\left\{S^{2}\right\}$ corresponds an Abelian $A_{4}$, of type $(1,1)$, containing $k_{2}, k_{2}{ }^{1}, k_{2}{ }^{2}$. Now with respect to $k_{2}{ }^{i}(i=1,2), K$ is relative Abelian of type $(1,1)$ : The three relative fields are $k_{4}{ }^{i}$ (counted twice) and $A_{4}$. Then, by (26a), if $F \equiv k_{2}{ }^{i}$, and $m=1, f=2$,

whence

But

$$
\zeta_{k_{2}}^{2} \zeta_{K}=\zeta_{k_{i}} \zeta_{A_{4}},
$$

$$
\zeta_{k_{2}^{1}} \zeta_{k_{2}^{2}} \zeta_{K}=\zeta_{A_{1}} \zeta_{k_{1}^{1}} \zeta_{k_{4}^{2}} \text {. }
$$

$$
\zeta^{2} \zeta_{A_{4}}=\zeta_{k_{2}} \zeta_{k_{2}} \zeta_{k_{2}^{2}}^{2}
$$

therefore, finally,

$$
\zeta^{2} \zeta_{K}=\zeta_{k_{2}} \zeta_{k_{1} \zeta^{2}} \zeta_{k_{4}{ }^{2}} \text {. }
$$

\section{CoMparison OF REgUlators}

After the fundamental Dirichlet-Dedekind expression, $h$, the class number of a field $k$, may be evaluated thus:

where

$$
\begin{aligned}
h \mu & =\lim _{s=1}(s-1) \zeta_{k}(s), \\
\mu & =\frac{2^{r_{1}+r_{2}} \pi^{r_{2}} R}{w\left|d^{1 / 2}\right|},
\end{aligned}
$$

$$
\begin{aligned}
& r_{1}=\text { number of real fields conjugate to } k, \\
& r_{2}=\text { number of pairs of imaginary fields conjugate to } k, \\
& w=\text { number of roots of unity in } k, \\
& R=\text { regulator in } k_{*}^{*}
\end{aligned}
$$

Let us return to the (absolute) Galois field $K$ with group $G_{m}$ as defined in $\S 1$. We shall assume in what follows that $K$ is real; we see then that

\footnotetext{
* See, for example, Hecke, Die Theorie der Algebraischen Zahlen, p. 156.
} 


$$
\mu(K)=\frac{2^{m-1} R(K)}{[d(K)]^{1 / 2}}, \quad \mu\left(k_{l}\right)=\frac{2^{l-1} R\left(k_{l}\right)}{\left[d\left(k_{l}\right)\right]^{1 / 2}}, \quad \mu\left(k_{a}\right)=\frac{2^{a-1} R\left(k_{a}\right)}{\left[d\left(k_{a}\right)\right]^{1 / 2}} .
$$

Hence, by (1), (2), and (37)

$$
\frac{h(K)}{h^{a}\left(k_{l}\right) h\left(k_{a}\right)}=\frac{R^{a}\left(k_{l}\right) R\left(k_{a}\right)}{R(K)} .
$$

We shall now consider the quotient of regulators in the right member of (38).

Let $\epsilon_{1}, \cdots, \epsilon_{a-1}$ be a set of fundamental units in $k_{a} ; \eta_{1}, \cdots, \eta_{l-1}$ a set of fundamental units in $k_{l}$. It will be convenient to employ the following abbreviations:

$$
\begin{array}{ll}
T_{i} E_{j}=\log \left|T_{i \epsilon_{j}}\right| & (i=1, \cdots, a ; j=1, \cdots, a-1), \\
S_{i} H_{j}=\log \left|S_{i} \eta_{j}\right| & (i=1, \cdots, l ; j=1, \cdots, l-1),
\end{array}
$$

where, as in $\$ 2, G_{l}=\left(S_{1}, \cdots, S_{l}\right), G_{a}=\left(T_{1}, \ldots, T_{a}\right), S_{1}=T_{1}=I$. We construct the set of $a$ distinct elements $T_{i} S_{2} T_{i}^{-1}(i=1, \cdots, a)$. It will be convenient to denote the members of this set by $U_{1}, \ldots, U_{a}$.

We now consider the set of $(m-1)$ units $\epsilon_{i}, U_{\alpha} \eta_{j}(i=1, \ldots, a-1$; $j=1, \cdots, l-1 ; \alpha=1, \cdots, a)$. Our object is to prove that at least when $l$ is a prime, they form a set of independent units in $K$. Since much of the work goes through in the general case, we shall make no assumption about the nature of $l$ until it is necessary. The regulator of the units in question will be called $R_{0}$; it will be formed thus:

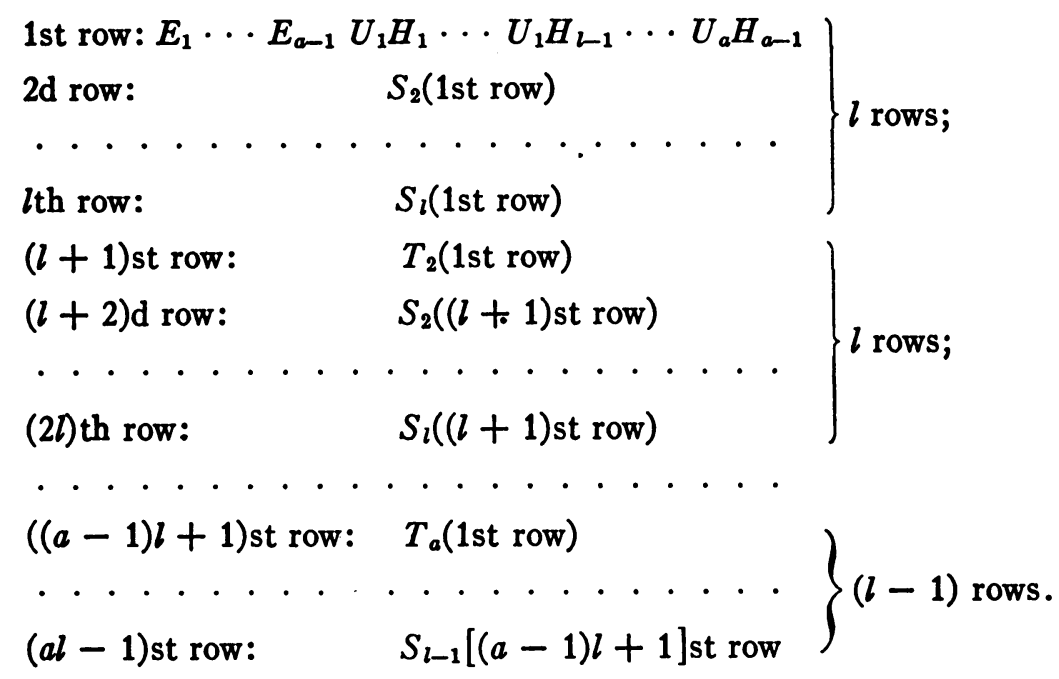

A first simplification of this determinant can be effected by noticing that (i) $\quad S E_{i}=E_{i}$, (ii) $S_{1} H_{j}+\cdots+S_{l} H_{i}=0$. 
Let us add the 1st, $2 \mathrm{~d}, \ldots,(l-1)$ st rows to the $l$ th row; it will become

$$
l E_{1} \cdots l E_{a-1} 00 \cdots(a l-a \text { zeros }) .
$$

Similarly, if we add the $(l+1)$ st, $\ldots,(2 l-1)$ st rows to the $(2 l)$ th row, it will become

$$
l T_{2} E_{1} \cdots l T_{2} E_{a-1} 00 \cdots(a l-a \text { zeros }) .
$$

And so on. It is clear that $R_{0}$ becomes

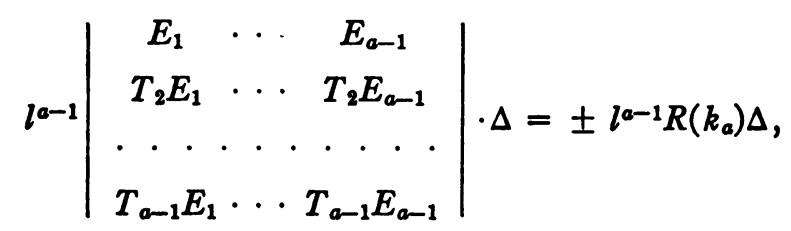

where

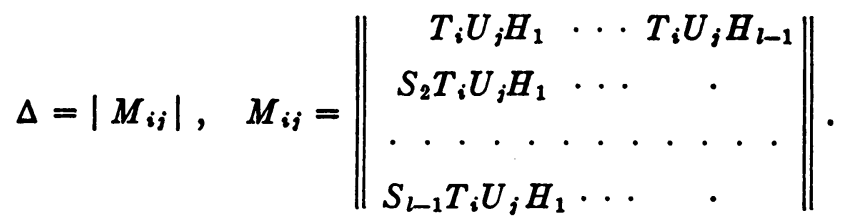

Now from the definition of $U_{i}$ it is clear that the set of matrices $M_{i j}(i$ fixed; $j=1, \cdots, a)$ is identical except for order with the set $M_{1 j}$. Furthermore the set of permutations

$$
\left(\begin{array}{ll}
M_{11} & M_{12} \cdots M_{1 a} \\
M_{i 1} & M_{i 2} \cdots M_{i a}
\end{array}\right)
$$

form a group isomorphic with $G_{a}$.

Let us write $M_{i j}=N_{i j} \bar{R}$,

$$
\bar{R}=\left\|S_{i} H_{j}\right\| \quad(i, j=1, \cdots, l-1) .
$$

Then

$$
\begin{aligned}
\Delta & =\left|N_{i j}\right| \cdot|\bar{R}|^{a} \\
& = \pm\left|N_{i j}\right| \cdot R^{a}\left(k_{l}\right) .
\end{aligned}
$$

The determinant $\left|N_{i j}\right|$ is a generalization of the class of determinants known as circulants. When $G_{a}$ is Abelian the determinant may be transformed into a product of determinants of the $(l-1)$ st order; this is a direct generalization of a known result.*

Assuming then that $G_{a}$ is Abelian, let $\chi_{\alpha}(T)(\alpha=1, \ldots, a)$ represent the

\footnotetext{
* Burnside, Messenger of Mathematics, vol. 23, p. 112.
} 
$a$ characters of the group.* Let

then

$$
X_{0}=\left|x_{i}\left(T_{j}\right)\right| \quad(i, j=1, \cdots, a) ;
$$

Therefore

$$
\begin{aligned}
\left|X_{0}\right|^{2} & =\left|\chi_{i}\left(T_{j}\right)\right| \cdot\left|\chi_{i}\left(T_{j}^{-1}\right)^{\prime}\right|=\left|\sum_{\alpha} \chi_{\alpha}\left(T_{i}\right) \chi_{\alpha}\left(T_{j}^{-1}\right)\right| \\
& =\left|\sum_{\alpha} \chi_{\alpha}\left(T_{i} T_{j}^{-1}\right)\right|=a^{a} .
\end{aligned}
$$

$$
\left|X_{0}\right|=a^{a / 2} \neq 0 \text {. }
$$

Let now $X=\left|\chi_{i}\left(T_{j}\right) E\right|$, where $E$ is the $(l-1)$-rowed unit matrix; clearly, $X=X_{0}^{l-1}$. Therefore

$$
X \neq 0 \text {. }
$$

Returning to $\left|N_{i j}\right|$, we have

$$
\begin{aligned}
\left|N_{i j}\right| \cdot X & =\left|N_{i j}\right| \cdot\left|\chi_{i}\left(T_{j}\right) E\right|=\left|\sum_{\alpha} \chi_{i}\left(T_{\alpha}\right) N_{j \alpha}\right| \\
& =\left|\sum_{\alpha} \chi_{i}\left(T_{\alpha} T_{j}^{-1}\right) N_{1 \alpha}\right|=\left|\chi_{i}\left(T_{i}^{-1}\right) \sum_{\alpha} \chi_{i}\left(T_{\alpha}\right) N_{1 \alpha}\right| \\
& =\left|\chi_{i}\left(T_{j}^{-1}\right) E\right| \cdot \prod_{i}\left|\sum_{\alpha} \chi_{i}\left(T_{\alpha}\right) N_{1 \alpha}\right| .
\end{aligned}
$$

Therefore, by (41),

$$
\left|N_{i j}\right|=\prod_{i}\left|\sum_{\alpha} \chi_{i}\left(T_{\alpha}\right) N_{1 \alpha}\right|
$$

in absolute value.

Now exactly the same result obtains when $N_{1 \alpha}$ is an ordinary (rather than a matric) quantity. We see then that a determinant like $\left|N_{i j}\right|$ may be reduced to a product of determinants of order $l-1$ by treating its matric elements as if they were ordinary numbers.

We suppose in the following that $G_{m}$ is a "congruence group, modulo $l$," that is, a group that may be defined as the set of transformations $\dagger$

$$
x^{\prime} \equiv \alpha x+\beta
$$

Let $r$ appertain to the index $a, \bmod l$; then, if we define a matrix $A$ of order $(l-1)$ by

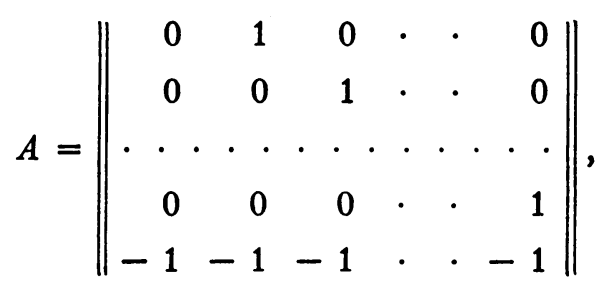

* Weber, loc. cit., p. 49.

† Fricke, loc. cit., vol. 1, p. $443 \mathrm{ff}$. 
it is easily seen that the set of matrices $N_{1 \alpha}$ is now replaced by $A^{i}(i=0, \ldots$, $a-1)$. It should be noticed that

$$
A^{l}=E, E+A+\cdots+A^{l-1}=0 .
$$

Also, since $G_{\alpha}$ is now cyclic, its characters may be expressed in terms of $\rho$, a primitive root of unity of index $a$.

From the above it readily follows that (42) becomes

$$
\left|N_{i j}\right|=\prod_{i}\left|\sum_{j} \rho^{i j} A^{r^{j}}\right|
$$

The determinants in the right member may be evaluated by substituting for $A$ a primitive $l$ th root of unity, $\zeta$. Now, $\sum_{i} \rho^{i j} \zeta{ }^{j}$ is not zero; this follows from Kronecker's theorem on the irreducibility of the cyclotomic equation. We may then assert that $\left|N_{i j}\right| \neq 0$. Therefore, by (39) and (40), $R_{0}$ is not zero, so that the statement made at the beginning of this section is substantiated: $\epsilon_{i}, U_{\alpha} \eta_{j}$ form a set of independent units in $K$.

Explicit results may be obtained very easily in two extreme cases: (I) $a=l-1$; (II) $a=2$.

(I) $a=l-1$. To evaluate the right member of (45) we note that $\sum_{j=0}^{a-1} \rho^{i j} \zeta^{j}$ is now the familiar Lagrange resolvent generally denoted by $\left(\rho^{i}, \zeta\right)$. For the resolvent we have

$$
\left(\rho^{0}, \zeta\right)=-1 \text { and }\left(\rho^{i}, \zeta\right)\left(\rho^{-1}, \zeta\right)=(-1)^{i} l \quad(i=1, \cdots, l-2)
$$

Hence, in this case,

$$
\left|N_{i j}\right|=l^{(l-1)(l-2) / 2} \text { in absolu te value. }
$$

Therefore

$$
R_{0}=l^{(l+1)(l-2) / 2} R\left(k_{a}\right) R^{a}\left(k_{l}\right) .
$$

(II) $a=2 . \quad\left|N_{i j}\right|$ is here

$$
\left|\begin{array}{ll}
A & A^{-1} \\
A^{-1} & A
\end{array}\right|=\left|A^{2}-A^{-2}\right|=\left|A^{-2}\right| \cdot|A-E| \cdot|A+E| \cdot\left|A^{2}+E\right| \text {. }
$$

But, from (43), $|A|=1$.

Secondly,

$$
|A+E| \cdot\left|A^{2}+E\right| \cdots\left|A^{l-1}+E\right|=1,
$$

whence $|A+E|=1,\left|A^{2}+E\right|=1$.

Thirdly,

$$
|I-A| \cdot\left|I-A^{2}\right| \cdots\left|I-A^{l-1}\right|=|l|=l^{l-1} ;
$$


therefore

$$
|I-A|=l
$$

Finally, then,

$$
R_{0}=l^{2} R\left(k_{a}\right) R^{2}\left(k_{l}\right) .
$$

Returning to (46), we shall now show that the ratio of $R_{0}$ to $R(K)$ is a power $(\geqq 0)$ of $l$.

Assume $R_{0}>R(K)$; then there exist a positive integer $n$, and a unit $B$ of $K$, such that

$$
\sum_{1}^{l-2} b_{i} E+\sum_{1}^{l-1} \sum_{1}^{l-1} b_{i j} S^{i} H_{j}=n \log |B|
$$

where

$$
0 \leqq b_{i}<n, \quad 0 \leqq b_{i j}<n ;\left(b_{1}, \cdots, b_{l-2}, b_{11}, \cdots, b_{l-1, l-1}\right)=1 .
$$

Putting (47) in exponential form, applying $T^{u}$, and reverting to the logarithmic form $\left(T S T^{-1}=S^{u}\right)$,

$$
\sum b_{i} T^{u} E_{i}+\sum \sum b_{i j} S^{i} u H_{j}=n \log \left|T^{u} B\right| \quad(u=1, \cdots, l-2) .
$$

Adding (47) to the $(l-2)$ equations (48), we have

$$
\sum_{i, j, u=1}^{l-1} b_{i j} S^{i r^{u}} H_{j}=n \log \left|B \cdot T B \cdots T^{l-2} B\right|=n \log |\alpha|,
$$

$\alpha$ a unit in $k_{l}$.

Now

so that (49) becomes

$$
\begin{aligned}
\sum_{u} S^{i r^{u}} H_{j} & =\sum_{u} S^{i u} H_{j} \\
& \left.=\sum_{u} S^{u} H_{j} \text { (since } 1 \leqq i \leqq l-1\right) \\
& =-H_{\cdot j},
\end{aligned}
$$

that is

$$
-\sum \sum b_{i j} H_{j}=n \log |\alpha|
$$

$$
-\sum_{i}\left(\sum b_{i j}\right) H_{j}=n \log |\alpha|
$$

But since the $\eta_{j}$ form a fundamental set in $k_{l}$, this last equation implies

$$
n \mid \sum_{i} b_{i j} \text {. }
$$

We return again to (47) and begin by applying $S^{-1}$ : 


$$
\sum b_{i} E_{i}+\sum \sum b_{i j} S^{i-1} H_{j}=n \log \left|S^{-1} B\right| .
$$

The double summation can be transformed thus:

$$
\begin{aligned}
\sum_{i, j=1}^{l-1} b_{i j} S^{i-1} H_{j} & =\sum_{j} b_{i j} H_{j}+\sum_{j} \sum_{i=2}^{l-1} b_{i j} S^{i-1} H_{j} \\
& =-\sum_{i} \sum_{j} b_{1 j} S^{i} H_{j}+\sum_{j} \sum_{i=1}^{l-2} b_{i+1, j} S^{i} H_{j} \\
& =\sum_{i} \sum_{i} \bar{b}_{i j} S^{i} H_{j},
\end{aligned}
$$

where

$$
\begin{array}{ll}
\bar{b}_{j i}=b_{i+1, j}-b_{1 j} & \text { for } 1 \leqq i \leqq l-2, \\
\bar{b}_{i j}=-b_{1 j} & \text { for } i=l-1 .
\end{array}
$$

(51) may now be written as

$$
\sum b_{i} E_{i}+\sum \sum \bar{b}_{i j} S^{i} H_{j}=n \log |\bar{B}| \quad\left(\bar{B}=S^{-1} B\right) .
$$

But (52) is exactly like (47); therefore from (50)

$$
n \mid \sum_{i} \bar{b}_{i j} \text {. }
$$

(Note that in proving (50) no use is made of the auxiliary relations in (47).) This may be written, in terms of $b_{i j}$, in the following way:

$$
n \mid\left(\sum_{i} b_{i j}-l b_{1 j}\right) \text {. }
$$

In the same way, we may extend (53) to

$$
n \mid\left(\sum_{i} b_{i j}-l b_{u i}\right) \quad(u=1, \cdots, l-1)
$$

or, making use of (50),

$$
n \mid l b_{i j}
$$$$
(i, j=1, \cdots, l-1)
$$

that is, $n \mid l \cdot\left(b_{11}, b_{12}, \cdots, b_{l-1, l-1}\right)$.

Put $\sigma=\left(b_{11}, b_{12}, \cdots, b_{l-1, l-1}\right)$, and assume

$$
(n, \sigma)=\tau>1 \text {. }
$$

Then (47) may be written

$$
\sum b_{i} E_{i}=\tau \log \left|B_{1}\right|,
$$

or

$$
\epsilon=B_{1}{ }^{T}, \quad \epsilon=\epsilon_{1}^{b_{1}} \cdots \epsilon_{l-2}^{b_{l-2}} .
$$


By one of the conditions in $(47),\left(\tau, b_{1}, \ldots, b_{l-2}\right)=1$, so that $\epsilon$ is not the $\tau$ th power of a unit in $k_{a}$. It remains to show that $\epsilon=B_{1}^{\tau}$ is impossible for any $B_{1}$ of $K$. Since $k_{a}$ is a maximal proper subfield of $K, \tau$ must be a multiple of $l$. Hence we need only consider the possibility of

$$
\epsilon=B_{1}{ }^{l} \text {. }
$$

But as $K$ does not (under our assumptions) contain the $l$ th roots of unity, this equality cannot hold.

We see then that $\tau$ is unity, and therefore

$$
n \mid l \text {. }
$$

Taking this in conjunction with (47), we see that our assertion on the ratio of $R_{0}$ to $R(K)$ is proved.

It is natural to enquire as to the number of independent relations like (47) actually existing in a field $K$. An exact answer apparently entails great difficulties; however it is easy to determine the maximum number of such relations. Making use of (50), this number is seen to be $(l-1)(l-2)$. Therefore, we have at once

$$
\frac{R_{0}}{R(K)} \mid l^{(l-2)(l-1)}
$$

(46) may then be written

$$
l^{n} R(K)=l^{(l+1)(l-2) / 2} R\left(k_{a}\right) R^{l-1}\left(k_{l}\right) \quad(0 \leqq \lambda \leqq(l-1)(l-2)) .
$$

If (57) be compared with (36), we obtain the following class-number relationship $(a=l-1)$ :

$$
h(K)=l^{(l+1)(l-2) / 2-\lambda} h\left(k_{a}\right) h^{l-1}\left(k_{l}\right) .
$$

It is of course rather obvious that the methods of this section may be applied to the other types of fielàs considered above. It does not however seem possible (using only these methods) to go as far as (57). On the other hand, Pollaczek, in the paper referred to above, obtains interesting results in the Abelian case ( $\$ 5$ ).

Unversity of Pennsylvania, Philadelphia, Pa. 